\title{
Marine Bacteria Is the Cell Factory to Produce Bioactive Pigments: A Prospective Pigment Source in the Ocean
}

\author{
Palanivel Velmurugan ${ }^{1}$, Chidambaram Kulandaisamy Venil ${ }^{2}$, Arumugam Veera Ravi ${ }^{1 *}$ and \\ Laurent Dufossé ${ }^{3 *}$ \\ ' Department of Biotechnology, Anna University Regional Campus, Coimbatore, India, ${ }^{2}$ Department of Biotechnology, \\ Alagappa University-Science Campus, Karaikudi, India, ${ }^{3} \mathrm{CHEMBIOPRO}$ Chimie et Biotechnologie des Produits Naturels, \\ ESIROI Département Agroalimentaire, Université de la Réunion, Sainte-Clotilde, France
}

OPEN ACCESS

Edited by:

Cristobal N. Aguilar,

Universidad Autónoma de

Coahuila, Mexico

Reviewed by:

Guadalupe Virginia Nevárez-Moorillón, Autonomous University of

Chihuahua, Mexico

Sabu Abdulhameed,

Kannur University, India

Débora A. Campos,

Escola Superior de Biotecnologia -

Universidade Católica

Portuguesa, Portugal

*Correspondence:

Arumugam Veera Ravi aveeraravi@rediffmail.com

Laurent Dufossé

laurent.dufosse@univ-reunion.fr

Specialty section:

This article was submitted to

Sustainable Food Processing,

a section of the journal

Frontiers in Sustainable Food Systems

Received: 31 July 2020 Accepted: 26 October 2020 Published: 26 November 2020

Citation:

Velmurugan $P$, Venil $C K$, Veera Ravi A and Dufossé L (2020) Marine Bacteria Is the Cell Factory to Produce

Bioactive Pigments: A Prospective Pigment Source in the Ocean. Front. Sustain. Food Syst. 4:589655. doi: 10.3389/fsufs.2020.589655
The course of investigations of bioactive compounds like bacterial pigments from the marine environment has greatly expanded in the recent decades. Despite the huge concern in secluding and collecting marine bacteria, microbial metabolites are progressively alluring to science due to their wide ranging applications in various fields, particularly those with distinctive color pigments. This review is a short appraisal of the studies undertaken over the past 5 years on the bacterial pigments sourced from the marine environment. Herein, we have reviewed the potential of different bacterial species isolated from marine environment in diverse studies that are producing bioactive pigments that have potential commercial applications.

Keywords: production, pigments, bioactive, cell factory, bacteria, marine

\section{INTRODUCTION}

Marine environments are one of the most important eco-systems of our earth that are yet to be explored to understand their full potential. They contain immeasurable and uncountable undiscovered mysterious black boxes holding colossal information that is to be decoded scientifically and exploited for the benefit of mankind. Marine environments are home to a multitude of organisms ranging from whales that are to a height of a building to microorganisms that are nano-scale life forms. The marine microorganisms are renowned to maintain and regulate the Bio-geo chemical cycle in the ocean environment and the interactions of the microbial community in the ocean to maintain such a biogeochemical cycle extraordinarily; metabolomics studies by profiling the variety of molecules reveal that the organisms produce to interact (Sogin et al., 2019). More than 6,000 and 10,000 species of prokaryotes and protists have been described so far (Aryal et al., 2015); yet the ocean hides an even more complex diversity of microorganisms that we are yet to fathom by appropriate investigations and explorations. Currently, the marine bacteria are being explored for their production of clinically and industrially important secondary metabolites; the pigments produced by marine bacteria as a result of quorum sensing are of current interest due to their anti-microbial, anti-cancer, photoprotective, anti-parasitic, and immunosuppressive activities (Ramesh et al., 2019). The modification of current natural compounds to synthetic compounds seems to be slow compared to the exponential evolution of resistant organisms which forces our hands to look for alternatives where secondary metabolites from marine bacteria seem to be the answer (Andryukov et al., 2019). Bacterial 
pigments are also used as fluorescence-based indicators for labeling antibodies, screen several reactions, and also to cure the damage caused by free radicals (DeLange and Glazer, 1989). The marine bacteria produce various pigments like carotene, melanin, phenazine, pyrrole, violacein, and quinones (Figure 1). This review evaluates the advancements that were made in the studies of marine bacterial pigments in the past 5 years and their prospective applications in various fields (Table $\mathbf{1}$ ).

\section{PHENAZINE}

Phenazines are redox-active, small nitrogen-containing aromatic compounds produced by a diverse range of bacterial genera, including Streptomyces (terrestrial), Pseudomonas (ubiquitous), Actinomycetes (terrestrial and aquatic), Pelagibacter (aquatic), and Vibrio (aquatic), under the control of quorum sensing and also a nitrogenous aromatic compounds of reverse redox potential converting molecular oxygen into toxic reactive oxygen species and are used in a broad spectrum of antibiotics, antiviral, insecticidal agent, anti-cancerous, antiprotozoal agents (Pierson and Pierson, 2010; Soliev et al., 2011; Guttenberger et al., 2017). Phenazine also functions as a respiratory pigment which is pyocyanin (blue) and Pseudomonas sp., has been highly researched (Figure 1). The biosynthesis of phenazine is catalyzed by five enzymes PhzE, PhzD, PhzF, PhzB, PhzG where PhzE converts chorismic acid to 2-amino-2-deoxyisochorismic acid (ADIC) by diffusion of NH3 from GATase1 to active MST domain of the enzyme by ligand binding and end with $\Psi$ face ofC2 chorismate and converted to DHHA by PhzD by acid/base catalytic system and is isomerized by $\mathrm{PhzF}$ by utilizing glutamate and catalyze by proton shift into AOCHC; but it is still unstable by forming aminoketone $\mathrm{AOCHC}$ and goes into a second condensation reaction of AOCHC into a secondary molecular structure which is accelerated by $\mathrm{PhzB}$ and by conjugation HHPDC is produced which is unstable and undergoes oxidative decarboxylation resulting in THPCA which is a PhzG substrate producing PCA and if PhzG binds to HHPDC oxidation takes place producing PDC the two final core phenazine compounds (Blankenfeldt and Parsons, 2014). Liang et al. (2017) isolated six phenazines from Streptomyces sp., 182SMLY with three new phenazine compound (-)- streptophenazines $\mathrm{M}-\mathrm{O}$ and three already known phenazine 1-carbomethoxyphenazine; (-)streptophenazines A; streptophenazines B where it was tested against the proliferation of glioma cell lines but didn't have any inhibitory effect on proliferating glioma cells and it was also screened against $E$. coli and MRSA, but only streptophenazines $B$ was effective against MRSA compared to control norfloxacin which inhibited both E. coli and MRSA. Phenazine compound pyocyanin has been isolated from marine $P$. aeruginosa by Li et al. (2018), and demonstrated to be acting as an antichlamydial agent with a dose of IC50 $(0.02 \mu \mathrm{M})$ inhibiting its infectivity by directly targeting Elementary body (EB) but low doses didn't seem to increase the host ROS. The IC50 dose of pyocyanin did not show any immune suppressant activity and performed equally to the IC50 dose of Tetracycline against MRSA supporting the clinical use of pyocyanin for treatment. Patil et al. (2016a,b), reported that GS-33 marine P. aeruginosa produced Phenazine-1- carboxylic acid which inhibited the charcoal root rot caused by M. phaseolina, promotes plant growth, and even conferred resistance of plants toward saline conditions by producing $\mathrm{NH} 3$ and solubilizing phosphate. The GS-33 isolate PCA was also reported to inhibit skin melanoma in low doses in human cell melanoma cell lines SK-MEL-2 and when combined with the commercial SPF lotions showed a synergistic increase of around 10-30\% UV-B protection. Cytotoxic studies revealed that concentration up to $100 \mathrm{ppm}$ PCA was safe which showed hemolysis around permissible levels. The limited commercial availability of substituted phenazines indicates that their synthesis presents a challenge for the synthetic chemist. To date, no efficient and generally applicable synthesis of substituted phenazines exists (Laursen and Nielsen, 2004).

\section{CAROTENE}

Carotene is synthesized mainly by plants followed by other microorganisms like filamentous fungi, yeast, and bacteria which have been reported to display Pro-vitamin Activity and displays strong antioxidant activity. Carotene is used as food colorants, cosmetics, and in feed industries where their color ranges from yellow to red (Da Costa Cardoso et al., 2017). Carotene is a C40 Polyunsaturated hydrocarbon compound a resultant of condensation of C20 compounds (Liang et al., 2006). The biosynthesis of carotene takes place by two core terpenoids Isopentenyl pyrophosphate and dimethylallyl pyrophosphate which is synthesized from the MEP pathway and DXP pathway where the IPP and DMAPP are condensed into GGPP and FPP and converted to phytoene by phytoene synthase and metabolized into lycopene by lycopene synthase and finally to $\beta$-carotene by Lycopene $\beta$ cyclase and further undergoes five intermediates to produce astaxanthin (Zhang et al., 2020). Afra et al. (2017) has reported the isolation of red-colored carotenoid pigment from marine Arthrobacter sp., G20 which displayed excellent anti-oxidant activity where its EC50 scavenging activity was equivalent to $\beta$-carotene and $\alpha$-tocopherol. Mild tumor-suppressive activities were observed in esophageal cancer cells but specific antiproliferative activities were observed in the KYSE30 cell line where the cells were found to be round a characteristic feature of apoptotic cells. But, the extracted carotene pigment didn't show any appreciable anti-microbial activity. Kallscheuer et al. (2019) reported two planctomycetes strain $R$. rubra LF2T and $R$. brasiliensis Gr7 producing three carotenoid compounds saproxanthin, flexixanthin, $2^{\prime}$-isopentenyldehydrosaproxanthin and established biosynthetic pathway for the production of carotenoids. Asker (2017) have testified the production of carotenoids astaxanthin, 2- hydroxyastaxanthin, and 2,2'dihydroxyastaxanthin by Brevundimonas sp. strain N-5 which was isolated and characterized by high-throughput sequencing. Hegazy et al. (2020) have demonstrated that haloarchea Natrialba sp., displayed antiproliferative and apoptotic activity against colon, breast, liver, and cervical cancer cells by interfering and inhibiting the MMP-9pathway and displayed anti-viral activity 


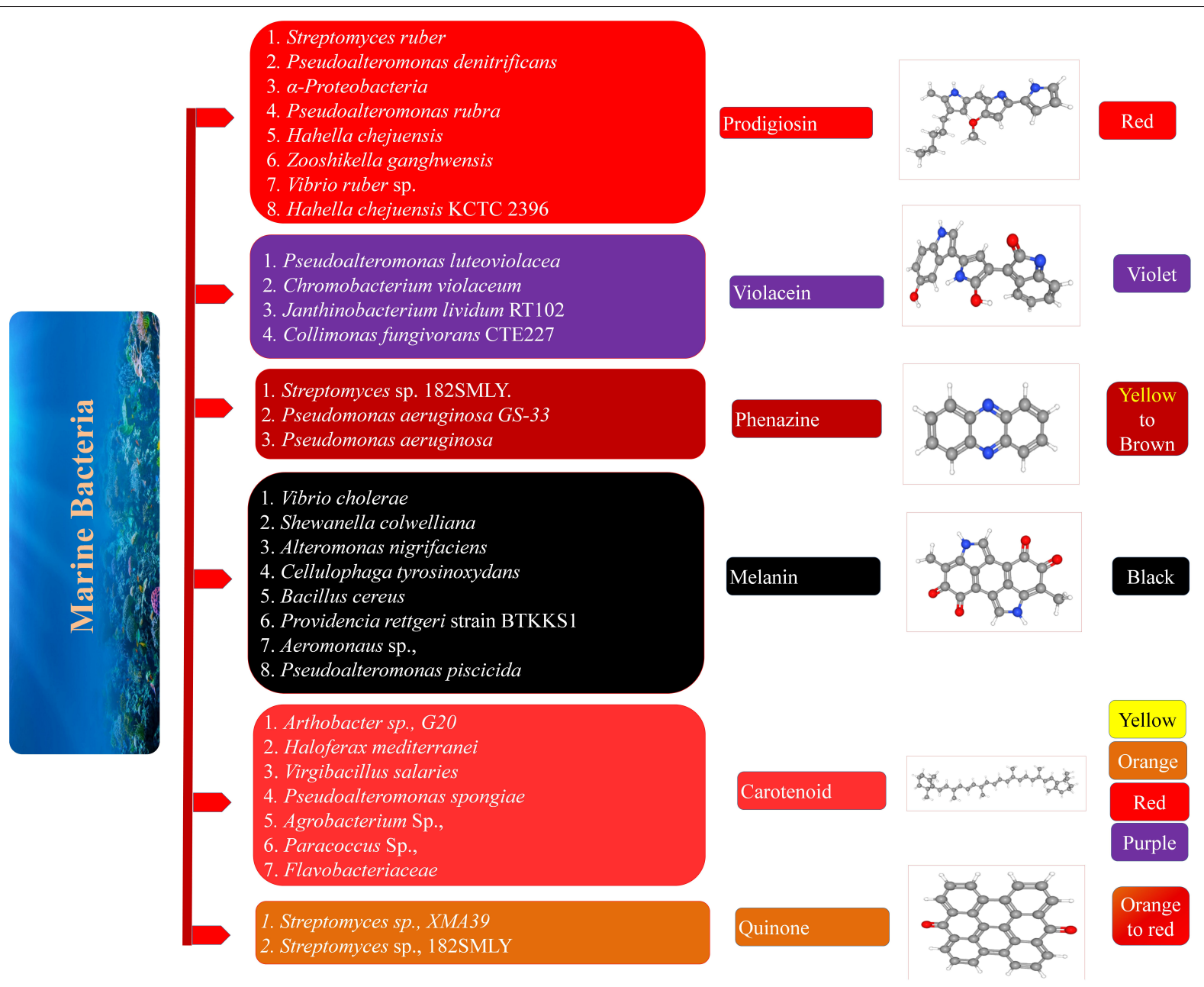

FIGURE 1 | Types of marine bacterial pigments.

by suppressing replication against HCV and HBV by inhibiting HCV NS5B polymerase and HBV-DNA dependent DNA polymerase. Earlier, synthetic $\beta, \beta$-carotene was produced on an industrial scale and used as a feed and food dye. Of the nearly 700 naturally occurring carotenoids, only a few are synthesized on an industrial scale. Among them are lycopene, canthaxanthin, astaxanthin, $\beta, \beta$-carotene, $\beta$-apo- $8^{\prime}$-carotenal, $\beta$-apo- $8^{\prime}$-carotene, and cytranaxanthin. Wittig reactions of Grignard compounds are used to obtain carotenoids (Alvarez et al., 2014; BogaczRadomska and Harasym, 2018).

\section{MELANIN}

Bacterial Melanin is a product of biosynthesis of Ltyrosine via the enzyme tyrosinase oxidizing it into L-3, 4dihydroxyphenylalanine which is further metabolized into dopachrome and finally converted to melanin by oxidoreduction where the color usually ranges from black-brown in color (ElNaggar and El-Ewasy, 2017). Traditional melanin nanoparticle synthesis usually takes around $12 \mathrm{~h}$, but Wang et al. (2020) demonstrated the production of melanin nanoparticle synthesis in bacteria within $30 \mathrm{~min}$ by controlling the growth of cell-free conditions by changing the salinity concentration of the medium and incubation period. This demonstrates the exciting potential of bacteria as melanin pigment factories and melanin has a broad range of usages such as anti-oxidative, anti-cancer, anti-bacterial, anti-viral properties also displaying thermal resistance, radiation damage by absorption of wide range electromagnetic spectrum, and even chemical resistance (Narsing Rao et al., 2017). Vijayan et al. (2017) isolated 156 sponge-associated bacteria out of which $14 \%$ displayed melanin production and its extraction from the sponge made the sponge colorless hinting the synergy between bacteria and the sponge for its photoprotective role and out of the $14 \%$ melanin-producing isolates $56 \%$ were observed to be Vibrio sp., and the remaining isolates were Providencia sp., Bacillus sp., S. algae, S. sciuri, P. maritimus, S. roseus, G. creatinolyticus; but the Vibrio sp., were selected for its high pigment production and the extracted pigment was observed for the photoprotective role from UV where highest efficiency was noted at $200 \mathrm{ppm}$ displaying $65 \%$ protective effect in mouse 
TABLE 1 | Microorganisms and its corresponding pigments.

\begin{tabular}{|c|c|c|}
\hline Organism & Pigment & References \\
\hline Shewanella algae & Melanin & Vijayan et al., 2017 \\
\hline Staphylococcus sciuri & Melanin & Vijayan et al., 2017 \\
\hline Planococcus maritimus & Melanin & Vijayan et al., 2017 \\
\hline Salinicoccus roseus & Melanin & Vijayan et al., 2017 \\
\hline Glutamicibacter Creatinolyticus & Melanin & Vijayan et al., 2017 \\
\hline Providencia sp., & Melanin & Vijayan et al., 2017 \\
\hline Bacillus sp., & Melanin & Vijayan et al., 2017 \\
\hline Pseudomonas stutzeri & Melanin & $\begin{array}{l}\text { Manirethan et al., 2018, } \\
2020\end{array}$ \\
\hline Pseudolateromonas lipolytica & Melanin & Kurian and Bhat, 2018 \\
\hline Chromobacterium violaceum & Violacein & Füller et al., 2016 \\
\hline Janithobacter lividum & Violacein & Füller et al., 2016 \\
\hline Psudoalteromonas amlolytica & Violacein & Wu et al., 2017 \\
\hline Streptomyces sp., XMA39 & Quinone & Liang et al., 2016 \\
\hline Streptomyces sp., 182SMLY & Quinone & Jiang et al., 2018 \\
\hline Zooshikella sp., & Prodigiosin & Ramesh et al., 2020 \\
\hline Streptomyces sp., & Prodigiosin & Ramesh et al., 2020 \\
\hline Vibryoruber DSM 14379 & Prodigiosin & Danevčič et al., 2016 \\
\hline Actinomyces sp., & Prodigiosin & Abdelfattah et al., 2019 \\
\hline Hahella sp., KA22 & Prodigiosin & Abdelfattah et al., 2019 \\
\hline $\begin{array}{l}\text { GS-33 marine Pseudomonas } \\
\text { aeruginosa }\end{array}$ & Phenazine & Patil et al., 2016a,b \\
\hline pseudomonas aeruginosa & Phenazine & Li et al., 2018 \\
\hline streptomyces sp., & Phenazine & Liang et al., 2017 \\
\hline Arthrobacter sp., G20 & Carotene & Afra et al., 2017 \\
\hline Rhodopirellula rubra LF2T & Carotene & Kallscheuer et al., 2019 \\
\hline Rubinisphaera brasiliensis Gr7 & Carotene & Kallscheuer et al., 2019 \\
\hline Natrialba sp., & Carotene & Hegazy et al., 2020 \\
\hline Brevundimonas sp., & Carotene & Asker, 2017 \\
\hline
\end{tabular}

fibroblast L-929 cells and brine shrimp compared to the control at $30 \%$ but levels higher than 200 pmm of melanin didn't show much efficiency or any cytotoxic effects up to $500 \mathrm{ppm}$ due to the transfer of extra energy to oxygen species producing Reactive oxygen species (Figure 1). Melanin extracted from bacteria has also been reported in heavy metal treatment, prevent fouling and formation of biofilm. Manirethan et al. (2018, 2020) used the melanin pigment extracted from gram-negative bacterium $P$. stutzeri for the absorption of heavy metals like copper, mercury, chromium, lead, and Arsenic where the absorption may be due to the presence of the $\mathrm{COOH}, \mathrm{NH}$, phenolic $\mathrm{OH}$ groups where maximum efficiency was absorbed in a $\mathrm{pH}$ ranging from mildly acidic to neutral. As a twist Melanin impregnated with copper and iron on its surface proved to be efficient in removing arsenic by chemisorption and it was also noted that melanin exhibited thermostability up to $120^{\circ} \mathrm{C}$ and showed peak absorption of arsenic at $\mathrm{pH}$ of 4-6 where XPS studies showed that arsenite before binding to $\mathrm{Cu}-, \mathrm{Fe}-$ Impregnated melanin oxidized to arsenate and finally removing the $\mathrm{Cu}$ and $\mathrm{Fe}$ by $\mathrm{Hcl}$ treatment for the recycling of melanin and again impregnating it with fresh $\mathrm{Cu}$ and $\mathrm{Fe}$ element for absorption which showed $99 \%$ efficiency to 4 absorption and desorption cycles. P. stutzeri BTCZ10 strain was reported to play a photoprotective role by displaying SPF value equivalent to sunscreen showed by Kurian and Bhat (2018). Zeng et al. (2017) reported the hyperpigmentation of pyomelanin which is a complex of polyphenolic heteropolymer produced under elevated temperature by marine hmgA mutant $P$. lipolytica displaying anti-fouling activity. Kiran et al. (2017) confirmed the anti-microbial activity against $S$. aureus, B. subtilis, E. coli, and $P$. aeruginosa displayed strong anti-biofilm activity against MDSA by nano-melanin synthesized by sonication of Pseudomonas sp., isolated from marine sponge T. citrine where the efficiency of nano-melanin was observed to be higher than the traditional melanin.

\section{PRODIGIOSIN}

Prodigiosin is blood-red colored compounds that display strong anti-microbial, cytotoxic, and immune suppressive activities by a range of mechanisms like decoupling $\mathrm{H} / \mathrm{Cl}$ - transporters modulating the $\mathrm{pH}$ of the cell and cleaves the DNA in the presence of copper (Huryn and Wipf, 2008). Prodigiosin biosynthesis undergoes a bifurcation pathway consisting of two systems a unique maltose-binding protein (MBP) and a common monolayer-protected cluster (MPC) biosynthesis. The 2-methyl-3-n-amyl-pyrrole (MAP) biosynthesis utilizes three genes pigB, pigD and pigE whereas the 4-methoxy-2,2' -bipyrrole5-carbaldehyde (MBC) biosynthesis uses seven genes pigA, pigFpigG, pigH, pigI, pigJ, pigL, and pigM and the products MAP and MBP are condensed together to form prodigiosin (Sakai-Kawada et al., 2019). Ramesh et al. (2020) isolated 17 different strains from south Andaman which produce pigments ranging from brown, yellow, red, and orange (Figure 1) where strain Zooshikella sp S2.1 and Streptomyces sp., BSE6.1 producing red pigment were confirmed to be prodigiosin was selected for further application in the use as food colorants and displayed effective antibacterial activity against $S$. aureus at concentrations of 150 to $400 \mu \mathrm{g} / \mathrm{mL}$. Marine V. ruber DSM 14379 isolated by Danevčič et al. (2016) exhibited the production of prodigiosin which showed strong bactericidal activity during the exponential phase of $B$. subtilis and bacteriostatic activity during stationary growth where it interferes with the cytoplasmic membrane and it also induces autolysin activity enhancing the killing of B. subtilis; however it was observed that the decrease of autolysin resulted in the abolished activity of prodigiosin. Abdelfattah et al. (2019), testified the antioxidant and antiinflammatory activity of prodigiosin extracted from Actinomyces sp., isolated from sponge $S$. mastoidea against $\mathrm{HCl} /$ ethanol gastric lesion by over-expressing $\mathrm{HO}-1$ resulting in elevated mucous production antioxidant activity, increasing HSP activity, apoptosis inhibition, and stabilizing cellular membrane thus preventing gastric injury. Prodigiosin has also been reported as an algicidal agent. Zhang et al. (2016) confirmed the algicidal activity of $P$. globosa by prodigiosin from Hahella sp., KA22 where prodigiosin inhibits photosynthesis by increasing ROS activity and finally necrotizes due to oxidative damage of the cells. 


\section{VIOLACEIN AND QUINONE}

Violacein an indole derivative is a purple-colored pigment exhibiting anti-tumor, anti-microbial properties mainly produced by C. violaceum and J. lividum (Masuelli et al., 2016). The biosynthesis of violacein starts from the oxidation of the precursor molecule tryptophan into indole-3-pyruvic acid by the flavoenzyme VioA, where VioB couples the two IPA imine molecule forming the intermediate Imine dimer and in the presence of Violacein biosynthesis protein VioE the dimer is converted to protodeoxyviolaceinic acid where VioD hydroxylates forming proto violacein ic acid and VioC hydroxylates and undergoes oxidative decarboxylation producing violacein (Durán et al., 2007; Füller et al., 2016). Wu et al. (2017) described a novel-violacein producing organism and characterized it which was isolated from the surface of seawater of the Arabian sea and by $16 \mathrm{~S}$ typing, the strains JW1T and JW3 were closely related to Pseudoalteromonas sp., and displayed independent lineage where there seem to be a display phylogenetic and chemotaxonomic differences, phenotypic properties and hence the name $P$. amylolytica was proposed. Currently, C. violaceum is being used as a bioassay strain for quorum sensing and quorum quenching activity for violaceum production to understand the QS or QQ activity of the desired strain (Liu et al., 2018; Balakrishnan et al., 2020; Singh et al., 2020). Quinone displays the antiviral, anti-cancer, anti-microbial and insecticidal activities where the color ranges from yellow to red (Soliev et al., 2011). Liang et al. (2016) reports the suppression of glioma cell lines by two polycyclic quinones N-acetyl-N-demethyl rapamycin and strepto anthraquinone A at IC50 extracted from marine Streptomyces sp., 182SMLY which induced apoptosis in glioma cells. $\mathrm{N}$-acetyl-N-demethylmayamycin was shown to be highly effective against MRSA. Quinones are also reported to display anti-fungal activities. Jiang et al. (2018) extracted medermycin-type naphthoquinones -strepoxepinmycin A to D and medermycin from Streptomyces sp., XMA39 where C and D showed cytotoxicity against HCT-116 and PC-3 cell lines and moderate inhibition of ROCK2 kinase was also observed. Collectively all the five compounds exhibited antagonistic activity against E. coli, S. aureus, and Candida sp. (Figure 1).

\section{FUTURE PERSPECTIVE}

Recently, a number of review papers have appeared in the literature, and they give an outline of all findings of the marine environment and its isolates. However, in this minireview, we focus exclusively on the active marine bacterial pigmented compounds and its potential applications have been

\section{REFERENCES}

Abdelfattah, M. S., Elmallah, M. I., Ebrahim, H. Y., Almeer, R. S., Eltanany, R. M., and Abdel Moneim, A. E. (2019). Prodigiosin from a marine spongeassociated actinomycete attenuate $\mathrm{HCl}$ /ethanol-induced gastric lesion via stated. The secondary metabolites of marine bacteria are gaining importance for its remarkable potential as anti-cancer, antimicrobial, anti-parasitic, insecticidal, anti-fowling, and antibiofilm properties. The continuous evolution of the resistance observed in pathogenic microbiota poses a significant threat corresponding to the time taken for the discovery of new drugs. The gap of altering the present product into a synthetic drug could be bridged by utilizing the full potential of the microorganism in the marine environment as the marine organisms for its survival produces secondary metabolites for competing with organisms in the community and when extracted it is of great potential. Due to the growth of high-throughput sequencing, metagenome, and the rapidly growing field of metabolomics study enables us to cultivate bacteria that are usually viable but non-cultivable. Despite the enormous difficulty in isolating and harvesting marine bacteria, significant progress has been achieved in this field, and investigations of bioactive compounds produced by these species are rapidly increasing. It has given us the set of tools to view from a distinct perspective helping us to understand the synergy that takes place in the marine ecosystem. Microbial pigments are of great interest due to its long fetching application from industry to pharmaceutical companies. Further large-scale studies are required to understand the interaction and evolution of these microorganisms that enable them to produce these pigments. Bacteria as pigment production units are of great interest due to its rapid production (or) fermentation of pigments. From the time consuming traditional synthesis of pigments bacterial pigment production could be achieved in a matter of minutes to hours by manipulating the cell-free condition, growth, and nutrients in the growth medium. This enables us to elect marine bacteria as a superior source factory for bioactive pigment production.

\section{AUTHOR CONTRIBUTIONS}

PV and CV: conceptualization, original draft preparation, and writing. LD: writing, review, and editing. AV: review. All authors contributed to the article and approved the submitted version.

\section{ACKNOWLEDGMENTS}

Financial support rendered to PV through RUSA 2.0 scheme in the form of Senior Postdoctoral fellowship [Grant No. F. 2451/2014-U, Policy (TN Multi-Gen), Department of Education, Government of India] is thankfully acknowledged. CV thanks the UGC for awarding the Dr. D. S. Kothari Post-doctoral Fellowship (BL/17-18/0479). 
Alvarez, R., Vaz, B., Gronemeyer, H., and de Lera, A. R. (2014). Functions, therapeutic applications, and synthesis of retinoids and carotenoids. Chem. Rev 114, 1-125. doi: 10.1021/cr400126u

Andryukov, B., Mikhailov, V., and Besednova, N. (2019). The biotechnological potential of secondary metabolites from marine bacteria. J. Marine Sci. Eng. 7:176. doi: 10.3390/jmse7060176

Aryal, S., Karki, G., and Pandey, S. (2015). Microbial diversity in freshwater and marine environment. Nepal J. Biotechnol. 3, 68-70. doi: 10.3126/njb.v3i1.14236

Asker, D. (2017). Isolation and characterization of a novel, highly selective astaxanthin-producing marine bacterium. J. Agric. Food Chem. 65, 9101-9109. doi: 10.1021/acs.jafc.7b03556

Balakrishnan, S., Ibrahim, K. S., Duraisamy, S., Sivaji, I., Kandasamy, S., Kumarasamy, A., et al. (2020). Antiquorum sensing and antibiofilm potential of biosynthesized silver nanoparticles of Myristica fragrans seed extract against MDR Salmonella enterica serovar Typhi isolates from asymptomatic typhoid carriers and typhoid patients. Environ. Sci. Pollut. Res. 27, 2844-2856. doi: 10.1007/s11356-019-07169-5

Blankenfeldt, W., and Parsons, J. F. (2014). The structural biology of phenazine biosynthesis. Curr. Opinion Struct. Biol. 29, 26-33. doi: 10.1016/j.sbi.2014.08.013

Bogacz-Radomska, L., and Harasym, J. (2018). $\beta$-Carotene-properties and production methods. Food Quality Safety 2, 69-74. doi: 10.1093/fqsafe/fyy004

Da Costa Cardoso, L. A., Kanno, K. Y. F., and Karp, S. G. (2017). Microbial production of carotenoids A review. Afr. J. Biotechnol. 16, 139-146. doi: 10.5897/AJB2016.15763

Danevčič, T., Borić Vezjak, M., Tabor, M., Zorec, M., and Stopar, D. (2016). Prodigiosin induces autolysins in actively grown Bacillus subtilis cells. Front. Microbiol. 7:27. doi: 10.3389/fmicb.2016.00027

DeLange, R. J., and Glazer, A. N. (1989). Phycoerythrin fluorescence-based assay for peroxy radicals: a screen for biologically relevant protective agents. Anal. Biochem. 177, 300-306. doi: 10.1016/0003-2697(89)90056-0

Durán, N., Justo, G. Z., Ferreira, C. V., Melo, P. S., Cordi, L., and Martins, D. (2007). Violacein: properties and biological activities. Biotechnol. Appl. Biochem. 48, 127-133. doi: 10.1042/BA20070115

El-Naggar, N. E. A., and El-Ewasy, S. M. (2017). Bioproduction, characterization, anticancer and antioxidant activities of extracellular melanin pigment produced by newly isolated microbial cell factories Streptomyces glaucescens NEAE-H. Sci. Reports 7:42129. doi: 10.1038/srep42129

Füller, J. J., Röpke, R., Krausze, J., Rennhack, K. E., Daniel, N. P., Blankenfeldt, W., et al. (2016). Biosynthesis of violacein, structure and function of 1Tryptophan oxidase VioA from Chromobacterium violaceum. J. Biol. Chem. 291, 20068-20084. doi: 10.1074/jbc.M116.741561

Guttenberger, N., Blankenfeldt, W., and Breinbauer, R. (2017). Recent developments in the isolation, biological function, biosynthesis, and synthesis of phenazine natural products. Bioorganic Med. Chem. 25, 6149-6166. doi: 10.1016/j.bmc.2017.01.002

Hegazy, G. E., Abu-Serie, M. M., Abo-Elela, G. M., Ghozlan, H., Sabry, S. A., Soliman, N. A., et al. (2020). In vitro dual (anticancer and antiviral) activity of the carotenoids produced by haloalkaliphilic archaeon Natrialba sp. M6. Sci. Reports 10, 1-14. doi: 10.1038/s41598-020-62663-y

Huryn, D. M., and Wipf, P. E. T. E. R. (2008). "Natural product chemistry and anticancer drug discovery," in Cancer Drug Design and Discovery, (Second Edition. Ed S, Neidle. (San Diego: Academic Press) (2014), 91-120.

Jiang, Y. J., Zhang, D. S., Zhang, H. J., Li, J. Q., Ding, W. J., Xu, C. D., et al. (2018). Medermycin-type naphthoquinones from the marine-derived Streptomyces sp. XMA39. J. Natural Products 81, 2120-2124. doi: 10.1021/acs.jnatprod.8b00544

Kallscheuer, N., Moreira, C., Airs, R., Llewellyn, C. A., Wiegand, S., Jogler, C., et al. (2019). Pink-and orange-pigmented Planctomycetes produce saproxanthintype carotenoids including a rare C45 carotenoid. Environ. Microbiol. Rep. 11, 741-748. doi: 10.1111/1758-2229.12796

Kiran, G. S., Jackson, S. A., Priyadharsini, S., Dobson, A. D., and Selvin, J. (2017). Synthesis of Nm-PHB (nanomelanin-polyhydroxy butyrate) nanocomposite film and its protective effect against biofilm-forming multi drug resistant Staphylococcus aureus. Sci. Reports 7, 1-13. doi: 10.1038/s41598-017-08816-y

Kurian, N. K., and Bhat, S. G. (2018). Data on the characterization of non-cytotoxic pyomelanin produced by marine Pseudomonas stutzeri BTCZ10 with cosmetological importance. Data brief 18, 1889-1894. doi: 10.1016/j.dib.2018.04.123
Laursen, J. B., and Nielsen, J. (2004). Phenazine natural products: biosynthesis, synthetic analogues, and biological activity. Chem. Rev. 104, 1663-1686. doi: $10.1021 / \mathrm{cr} 020473 \mathrm{j}$

Li, J. L., Yang, N., Huang, L., Chen, D., Zhao, Y., Tang, M. M., et al. (2018). Pyocyanin inhibits Chlamydia infection by disabling infectivity of the elementary body and disrupting intracellular growth. Antimicrob. Agents Chemother. 62:e2260-17. doi: 10.1128/AAC. 02260-17

Liang, C., Zhao, F., Wei, W., Wen, Z., and Qin, S. (2006). Carotenoid biosynthesis in cyanobacteria: structural and evolutionary scenarios based on comparative genomics. Int. J. Biol. Sci. 2:197. doi: 10.7150/ijbs. 2.197

Liang, Y., Chen, L., Ye, X., Anjum, K., Lian, X. Y., and Zhang, Z. (2017). New streptophenazines from marine Streptomyces sp. 182SMLY. Nat. Prod. Res. 31, 411-417. doi: 10.1080/14786419.2016.1169419

Liang, Y., Xie, X., Chen, L., Yan, S., Ye, X., Anjum, K., et al. (2016). Bioactive polycyclic quinones from marine Streptomyces sp. 182SMLY. Marine Drugs 14:10. doi: $10.3390 / \mathrm{md} 14010010$

Liu, L., Yan, Y., Feng, L., and Zhu, J. (2018). Quorum sensing asaI mutants affect spoilage phenotypes, motility, and biofilm formation in a marine fish isolate of Aeromonas salmonicida. Food Microbiol. 76, 40-51. doi: 10.1016/j.fm.2018.04.009

Manirethan, V., Raval, K., and Balakrishnan, R. M. (2020). Adsorptive removal of trivalent and pentavalent arsenic from aqueous solutions using iron and copper impregnated melanin extracted from the marine bacterium Pseudomonas stutzeri. Environ. Pollut. 257:113576. doi: 10.1016/j.envpol.2019. 113576

Manirethan, V., Raval, K., Rajan, R., Thaira, H., and Balakrishnan, R. M. (2018). Data on the removal of heavy metals from aqueous solution by adsorption using melanin nanopigment obtained from marine source: Pseudomonas stutzeri. Data brief 20, 178-189. doi: 10.1016/j.dib.2018.07.065

Masuelli, L., Pantanella, F., La Regina, G., Benvenuto, M., Fantini, M., Mattera, R., et al. (2016). Violacein, an indole-derived purple-colored natural pigment produced by Janthinobacteriumlividum, inhibits the growth of head and neck carcinoma cell lines both in vitro and in vivo. Tumor Biol. 37, 3705-3717. doi: 10.1007/s13277-015-4207-3

Narsing Rao, M. P., Xiao, M., and Li, W. J. (2017). Fungal and bacterial pigments: secondary metabolites with wide applications. Front. Microbiol. 8:1113. doi: 10.3389/fmicb.2017.01113

Patil, S., Paradeshi, J., and Chaudhari, B. (2016a). Suppression of charcoal rot in soybean by moderately halotolerant Pseudomonas aeruginosa GS-33 under saline conditions. J. Basic Microbiol. 56, 889-899. doi: 10.1002/jobm.201600008

Patil, S., Paradeshi, J., and Chaudhari, B. (2016b). Anti-melanoma and UV-B protective effect of microbial pigment produced by marine Pseudomonas aeruginosa GS-33. Nat. Prod. Res. 30, 2835-2839. doi: 10.1080/14786419.2016.1154057

Pierson, L. S., and Pierson, E. A. (2010). Metabolism and function of phenazines in bacteria: impacts on the behavior of bacteria in the environment and biotechnological processes. Appl. Microbiol. Biotechnol. 86, 1659-1670. doi: 10.1007/s00253-010-2509-3

Ramesh, C., Vinithkumar, N. V., and Kirubagaran, R. (2019). Marine pigmented bacteria: a prospective source of antibacterial compounds. J. Nat. Sci. Biol. Med. 10:104. doi: 10.4103/jnsbm.JNSBM_201_18

Ramesh, C., Vinithkumar, N. V., Kirubagaran, R., Venil, C. K., and Dufossé, L. (2020). Applications of prodigiosin extracted from marine red pigmented bacteria Zooshikella sp. and actinomycete streptomyces sp. Microorganisms 8:556. doi: 10.3390/microorganisms 8040556

Sakai-Kawada, F. E., Ip, C. G., Hagiwara, K. A., and Awaya, J. D. (2019). Biosynthesis and bioactivity of prodiginine analogues in marine bacteria, Pseudoalteromonas: a mini review. Front. Microbiol. 10:1715. doi: $10.3389 /$ fmicb. 2019.01715

Singh, A. A., Singh, A. K., and Nerurkar, A. (2020). Bacteria associated with marine macroorganisms as potential source of quorum-sensing antagonists. J. Basic Microbiol. 60, 799-808. doi: 10.1002/jobm.202000231

Sogin, E. M., Puskas, E., Dubilier, N., and Liebeke, M. (2019). Marine metabolomics: a method for nontargeted measurement of metabolites in seawater by gas chromatography-mass spectrometry. Msystems 4:e00638e00619. doi: 10.1128/mSystems.00638-19 
Soliev, A. B., Hosokawa, K., and Enomoto, K. (2011). Bioactive pigments from marine bacteria: applications and physiological roles. Evid. Based Complementary Alternat. Med. 2011:670349. doi: 10.1155/2011/670349

Vijayan, V., Jasmin, C., Anas, A., Kuttan, S. P., Vinothkumar, S., Subrayan, P. P., et al. (2017). Sponge-associated bacteria produce non-cytotoxic melanin which protects animal cells from photo-toxicity. Appl. Biochem. Biotechnol. 183, 396-411. doi: 10.1007/s12010-017-2453-0

Wang, Z., Tschirhart, T., Schultzhaus, Z., Kelly, E. E., Chen, A., Oh, E., et al. (2020). Melanin produced by the fast-growing marine bacterium vibrio natriegens through heterologous biosynthesis: characterization and application. Appl. Environ. Microbiol. 86:e02749-19. doi: 10.1128/AEM.02749-19

Wu, Y. H., Cheng, H., Xu, L., Jin, X. B., Wang, C. S., and Xu, X. W. (2017). Physiological and genomic features of a novel violaceinproducing bacterium isolated from surface seawater. PLOS ONE 12:e0179997. doi: 10.1371/journal.pone.0179997

Zeng, Z., Guo, X. P., Cai, X., Wang, P., Li, B., Yang, J. L., et al. (2017). Pyomelanin from i reduces biofouling. Microbial. Biotechnol. 10, 1718-1731. doi: $10.1111 / 1751-7915.12773$
Zhang, C., Chen, X., and Too, H. P. (2020). Microbial astaxanthin biosynthesis: recent achievements, challenges, and commercialization outlook. Appl. Microbiol. Biotechnol. 104, 5725-5737. doi: 10.1007/s00253-020-10648-2

Zhang, H., Peng, Y., Zhang, S., Cai, G., Li, Y., Yang, X., et al. (2016). Algicidal effects of prodigiosin on the harmful algae Phaeocystis globosa. Front. Microbiol. 7:602. doi: $10.3389 /$ fmicb. 2016.00602

Conflict of Interest: The authors declare that the research was conducted in the absence of any commercial or financial relationships that could be construed as a potential conflict of interest.

Copyright (C) 2020 Velmurugan, Venil, Veera Ravi and Dufossé. This is an openaccess article distributed under the terms of the Creative Commons Attribution License (CC BY). The use, distribution or reproduction in other forums is permitted, provided the original author(s) and the copyright owner(s) are credited and that the original publication in this journal is cited, in accordance with accepted academic practice. No use, distribution or reproduction is permitted which does not comply with these terms. 